\title{
Screening for Group A Streptococcal disease via Solid-State Nanopore Detection of PCR Amplicons
}

\author{
Simon King, ${ }^{1}$ Kyle Briggs, ${ }^{1}$ Robert Slinger ${ }^{2}$ and Vincent Tabard-Cossa ${ }^{1 *}$ \\ ${ }^{1}$ Department of Physics, University of Ottawa, Ottawa, Canada \\ ${ }^{2}$ Division of Microbiology and Infectious Disease, Children's Hospital of Eastern Ontario Research Institute, \\ Ottawa, Canada
}

Keywords: Solid-state nanopore, PCR, streptococcus pyogenes, diagnostics, single-molecule detection, nucleic acid

\begin{abstract}
Single molecule detection methods are becoming increasingly important for diagnostic applications. Practical Early detection of disease requires sensitivity down to the level of single copies of the targeted biomarkers. Of the candidate technologies that can address this need, solid-state nanopores show great promise as digital sensors for single-molecule detection. Here, we present work detailing the use of solid-state nanopores as downstream sensors for a PCR-based assay targeting group A streptococcus (strep A) which can be readily extended to detect any pathogen that can be identified with a short nucleic acid sequence. We demonstrate that with some simple modifications to the standard PCR reaction mixture, nanopores can be used to reliably identify strep A in clinical samples. We also discuss methodological best practices both for adapting PCR-based assays to solid-state nanopore readout as well as analytical approaches by which to decide on sample status.
\end{abstract}

Molecular methods for infectious disease diagnostic are gradually being introduced into clinical use, and this trend will only accelerate because of the Covid-19 pandemic. ${ }^{1-4}$ Many of these molecular tests typically employ a DNA amplification method to generate many copies of the target sequence and then rely on downstream optical sensors to detect a fluorescent signal generated by the presence of amplified nucleic acid products (i.e., amplicons) in the sample. However, the optical components and the use of dyes as labels, required for fluorescence-based detection, can impose some limitations on the cost, size, and robustness of the instrument or 
reagents. These factors may limit the use of molecular diagnostics at the point of care and in rural or remote settings. Alternative detection methodologies that can improve on speed, sensitivity and accuracy are being actively explored..$^{5,6}$

The use of solid-state nanopores for electrical detection of PCR products is one such strategy that can offer less costly, more miniaturizable, and more rugged alternative to optical sensors. Solid-state nanopores are molecular-sized openings in thin membranes that allow the passage of individual biomolecules immersed in a conductive solution. Under an applied voltage, a steady stream of ions transit the nanopore, creating a measurable electrical current that is disrupted by the passage of a single biomolecule. The characteristic electrical signature generated by a target molecule can enable target identification. ${ }^{7-9}$

To demonstrate this potential, a few solid-state nanopore sensing methods have been developed to detect specific nucleic acid (NA) targets. PNA probes have been used to bind to specific DNA sequences, ${ }^{7,8}$ and employed to detect the cystic-fibrosis mutation codon. ${ }^{9}$ Solid-state nanopores have also been used to detect polymorphic insertion or deletion mutations in DNA, which determine the virulence or drug-resistance of two different bacterial organisms, after cleavage with mutation site recognition enzymes. Conserved sequences of the HIV RNA genome have been detected using biotinylated ssDNA probes and monovalent streptavidin. ${ }^{10}$ DNA nanostructures were used to report the presence of a Zika virus gene by folding in its presence, as well as a microRNA biomarker for human lung cancer. ${ }^{11-13}$ RNA targets, including SARS-CoV2, were also quantified by reverse transcription to produce gene specific cDNA that could be counted without necessarily requiring amplification, though low-level of amplification steps improved the detection speed in cases of rare transcripts. ${ }^{14}$ Multiplexed detection of prostate cancer microRNA biomarkers has also been developed with an electro-optical nanopore platform. ${ }^{15}$ Using 
solid-state nanopores for direct detection of NA targets from clinical specimens is challenging, partly due to the complexity of most biofluids, which can clog the pore or severely complicate downstream data analysis by generating a variety of translocation signals, but also to the requirement for a high volume of statistics and a high degree of reproducibility needed for confidently obtaining useful clinical results. This requires employing many individual solid-state nanopore sensors, often of similar geometries, which is presently achieved by performing and repeating many measurements serially but would be accelerated if done in parallel with arrays of nanopores.

Here, we performed a proof-of-concept study to determine the feasibility of pathogen detection from clinical samples with solid-state nanopores. We targeted group A streptococcus (GAS) in throat swabs from children with pharyngitis. GAS is a very important pediatric bacterial pathogen, accounting for $20-30 \%$ of pharyngitis episodes in children and leading to many pediatric emergency department visits. ${ }^{16,17}$ The complications of untreated GAS pharyngitis include acute rheumatic fever, scarlet fever, tonsillopharyngeal abscess, and streptococcal toxic shock syndrome. ${ }^{18}$ Antibiotic therapy is used to prevent these severe complications and to reduce the duration of pharyngitis symptoms. Clinicians have traditionally depended on bacterial culture results from throat swabs to identify those infected with GAS. More recently, molecular tests for GAS that amplify organism DNA have been developed, ${ }^{19}$ but, as noted, such tests are often costly and devices used for testing may be less adapted for handheld operations at the point of need, limiting the widespread use of these tests. Nanopore-based diagnostics may be able to overcome these barriers, while potentially offering simple workflows and high throughput, and maintaining high sensitivity. 
In this work, we demonstrate the utility of solid-state nanopores as the sensing element in a screening test for the presence of Group A streptococcus (GAS) in throat swabs. We report the optimizations and workflow adaptations required to make PCR amplification compatible with downstream solid-state nanopore detection, present an analysis scheme based on statistical differences in capture rates to make a call on a clinical sample, and demonstrate that our homebrew PCR nanopore assay agrees well with gold standard cell-culture and qPCR-based tests results.

\section{RESULTS AND DISCUSSION}

In this study, screening for an infectious agent in a clinical sample is based on electrical identification of individual PCR amplicons using a solid-state nanopore sensor. To enable detection with an acceptably low level of false positives (specificity) and false negatives (sensitivity), the nanopore must distinguish target amplicons from all other molecules present in the sample or introduced during the amplification reaction, including small molecules, genomic DNA, proteins, and enzymes. The electrophoretic and electroosmotic forces in and around the pore can capture these unwanted molecules, and their collision and interaction with the pore as well as their full translocation can lead to measurable blockages in the ionic current signal that must be differentiated from the amplicon signal. The degree with which the nanopore sensor can report the detection of these molecules is a function of the pore properties (pore size, surface chemistry), operating condition (salt concentration, $\mathrm{pH}$, voltage), the properties of the off-target molecules in the sample (size, charge, and propensity to stick to and clog pores in a given set of operating conditions), and the response time of the amplifier-pore system. ${ }^{20-28}$ Consequently, we first performed control experiments to investigate the nanopore response to different PCR mixtures to identify adequate operating conditions for sensing amplicons. 


\section{Nanopore Detection from Commercial PCR mixtures}

Polymerase Chain Reaction (PCR) is the gold standard technique for amplifying nucleic acids ${ }^{29-31}$ and numerous kits are available commercially. These kits contain several reagents to optimize the amplification reaction following their protocol. Commercial kits are also often used with a downstream fluorescent optical readout and cannot a priori be assumed to be compatible with nanopore sensing. These off-the-shelf PCR master mix kits usually include components such as bovine serum albumin (BSA) and detergents (e.g., Tween20) used to stabilize enzymes during storage and minimize losses to walls of plastic tubes as well as additional proprietary or undisclosed ingredients to increase PCR yield and product lifetime. These molecules, which can be in relatively high concentrations, are known to produce signals when translocating a nanopore, but also to stick to the pore walls, increasing the low-frequency noise of the nanopore signal and often leading to clogs (reversible or permanent). ${ }^{32-35}$ Characterization of the signals caused by any PCR master-mix is therefore a critical control for any analysis of the amplification reaction with a nanopore sensor.

Figures 1a-c shows the results of a PCR mixture from a commercial kit sensed by a $6 \pm 2$ $\mathrm{nm}$ nanopore in $3.6 \mathrm{M} \mathrm{LiCl} \mathrm{pH} 8$ at $200 \mathrm{mV}$ (note the uncertainty in the pore size is due to the uncertainty in the effective membrane thickness, likely smaller than the nominal membrane thickness of $10 \mathrm{~nm}$ ). ${ }^{36,37}$ To perform this measurement, a commercial master mix (see Methods section) was added to a sensing salt to mimic the background signals expected of a PCR amplification reaction during nanopore sensing.

Figure $1 \mathrm{~b}$ shows a representative current trace of this experiment, sampled at $4.17 \mathrm{MS} / \mathrm{s}$ and low pass filtered at $500 \mathrm{kHz}$. The trace shows many different event shapes, including temporary clogs, and ends with the nanopore irreversibly clogging after about 10 minutes. Figure 
1c provides additional detail on the characteristics of the signals generated, showing a range of blockage depths from 1-3.5 nA and a wide distribution of passage times from 3-1000 $\mu \mathrm{s}$.

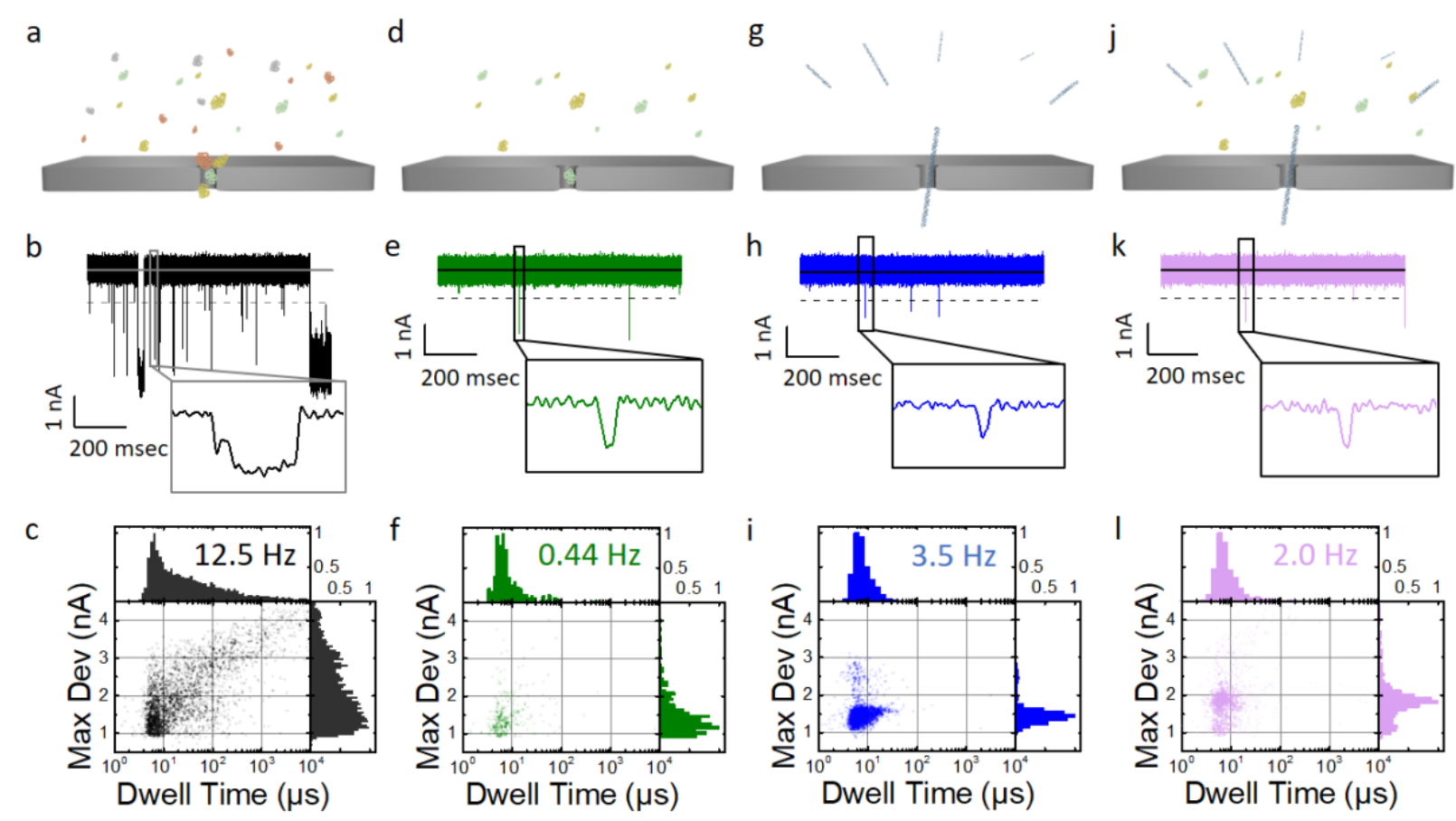

Figure 1: Nanopore sensing of different PCR mixtures. a) Schematic representation of a nanopore sensing a commercial PCR reaction mixture; b) 1-s current trace (black) of the commercial master mix; c) Maximum blockage depth of an event versus its passage time for the entire $\sim 10$ minutes dataset, comprised of 8,078 single-molecules events (black dots); d) Schematic of a nanopore sensing of a homebrew PCR mixture. e) 1-s current trace (green) of nanopore-optimized homebrew PCR master mix; f) Maximum blockage depth of an event versus its passage time for all 297 events identified during 10 minutes of recording with nanopore-optimized homebrew PCR mixture (green dots); g) Schematic representation of a nanopore sensing DNA amplicons; h) 1-s current trace (blue) of 100bp dsDNA at $75 \mathrm{nM}$; i) Maximum blockage depth of an event versus its passage time for all 2,779 events identified during 10 minutes with 100bp dsDNA (blue dots); j) Schematic of a nanopore sensing of a homebrew PCR-amplified reaction mixture; k) 1-s current trace of homebrew PCR-amplified reaction mixture containing homebrew mixture components and generated 100bp target amplicons from a GAS positive clinical sample; 1) Maximum blockage depth of an event versus its passage time for all 1,444 events identified during 10 minutes of recording with of PCR-amplified reaction mixture using homebrew PCR mixture containing 100bp target amplicons from a GAS positive clinical sample. Data for b) and c) acquired on the results of a PCR mixture from a commercial kit sensed by a $6 \pm 2 \mathrm{~nm}$ pore, while for i), k), 1) on a $5 \pm 1 \mathrm{~nm}$ diameter pore (with $7 \pm$ $2 \mathrm{~nm}$ effective thickness) and e), f), h) on the same pore that grew to $7 \pm 1 \mathrm{~nm}$ diameter pore (with $8 \pm 2$ $\mathrm{nm}$ effective thickness) many hours later, all at $200 \mathrm{mV}$ in $3.6 \mathrm{M} \mathrm{LiCl} \mathrm{pH8}$. Current traces displayed with a low-pass Bessel filter at $500 \mathrm{kHz}$ 
We attribute the large number of events and the complexity of the signals to the variety of molecules translocating the nanopore. The events observed can result from a complex combination of signals generated by the presence of detergents, fluorescent dyes, uracil-DNA glycosylase (UDG), and proteins included to improve stability and amplicon yield. Furthermore, under the high salt concentration condition used here, it is also possible for proteins to agglomerate and change structure. The data of Figure 1c revealed 8,078 single-molecule events in a $645 \mathrm{~s}$ of recording, exhibiting a high capture rate of molecules of $12.5 \mathrm{~Hz}$. A representative sampling of signal shapes attributed to the master mix and 100bp dsdDNA can be found in Supporting Section S1.

The passage of linear unfolded dsDNA molecules is expected to produce blockages of approximately $1.5 \pm 0.2 \mathrm{nA}$, depending on the value of the effective pore length. The presence of the amplicon population would therefore be difficult to discern from this commercial mixture. While the pore properties and operating conditions could be adjusted to potentially permit a better identification of amplicons from this commercial PCR mixture, the sensing conditions are far from optimized. Analysis is also complicated by the large number of events detected and the propensity of pore to clog that severely limits the nanopore lifetime and lowers experimental yield. To overcome these limitations, a homebrew PCR mixture was developed to be better suited to solidstate nanopore operation.

\section{Nanopore Detection from Homebrew PCR mixture}

To develop a PCR mixture compatible with nanopore detection (i.e., that minimizes the events from background and avoids frequent clogging), we optimized a reaction mixture starting from a minimal set of components. Details on this process can be found in Supporting Section S2. Characterization of this homebrew PCR mixture under optimized nanopore sensing conditions are 
shown in Figures 1d-f. In this negative control experiment, the solution contained the homebrew PCR reagents without any template (or genomic DNA), primer or amplicon present mixed in 3.6 M LiCl pH8 following 25 cycles of PCR amplification (see Methods section). The PCR reagents include Taq Polymerase at 25 units/mL, Tris- $\mathrm{HCl}$ at $10 \mathrm{mM}, \mathrm{KCl}$ at $50 \mathrm{mM}, \mathrm{MgCl}_{2}$ at $4 \mathrm{mM}$, and dNTPs at $200 \mu \mathrm{M}$.

The current trace (Figure 1e) using this optimized mixture reveals far fewer events compared to Figure $1 \mathrm{~b}$ for a similar sized pore $(\sim 5 \mathrm{~nm})$ at the same applied voltage of $200 \mathrm{mV}$. Additional current traces of the homebrew PCR mixture on additional pore sizes $6 \pm 2 \mathrm{~nm}$ are shown in Supporting Section S3. The solid black line represents the mean current, while the dashed line is the current blockage threshold at 7 standard deviations (SD) from the mean used to identify the beginning of a translocation event during our analysis. With these analysis settings, 297 events were detected in $679 \mathrm{~s}$, for a capture rate of $0.44 \mathrm{~Hz}$. The plot of the maximum blockage versus dwell time of an event (Figure 1f) shows a mean blockage depth of $1.2 \pm 0.3 \mathrm{nA}$ and passage time of $5 \pm 3 \mu \mathrm{s}$. While some events in this negative control experiments generate blockages that overlap with the estimated blockage depth $(\sim 1.5 \mathrm{nA})$ and mean passage time $(<10 \mu \mathrm{s})$ for single-file linear dsDNA in these conditions, this homebrew PCR mixture produces fast and relatively very few background signals, and most importantly is far less prone to clogging the nanopore when compared with the commercial kit. These features should greatly facilitate data collection and analysis when characterizing clinical samples.

Next, we characterized the signal from a positive control, wherein a $100 \mathrm{bp}$ dsDNA strand was added as a means by which to highlight the expected result of amplification of a target sequence from an infectious agent. The results of this positive control are shown in Figures 1g-i. Similar to the negative control, the presence of $75 \mathrm{nM}$ of $100 \mathrm{bp}$, rod-like, dsDNA produces events 
with a mean blockage of $1.4 \pm 0.2 \mathrm{nA}$. While these events are also translocating quite rapidly, they are more tightly distributed in passage time and blockage depth as compared to the events from the homebrew PCR mixture. Distinguishing an amplicon from a molecule in the homebrew PCR mixture on an individual event basis can be challenging, as the two populations show some overlap, although the frequency of events is much greater for this concentration of DNA, with 2,779 single-molecule events in a $779 \mathrm{~s}$ of recording. We note that the fast passage times of the short DNA molecules approaches the response time of our nanopore-amplifier system and we carefully set the analysis parameters (low-pass filter settings and detection threshold value) to provide appropriate signal-to-noise ratio to detect the blockage level of dsDNA while minimizing attenuation. For the average residence time of $\sim 2-5$ microseconds, a filter of $500 \mathrm{kHz}$ was chosen to minimize attenuation of signals $>2 \mu$ s.

Finally, to explore if the presence of amplicons in a homebrew PCR mixture can be discerned, we performed the full amplification reaction of a clinical GAS-positive sample using the homebrew PCR protocol and examined the result with a nanopore. Here, in addition to the homebrew PCR reagents and the amplicons, the sample contains genomic DNA and potentially other biomolecules from the clinical sample leftover from the assay workflow (see section below for assay workflow details). The results of this experiment performed on the same pore are shown in Figures $1 \mathrm{j}-1$. As expected, the populations of events observed resembles a superposition of both previously tested controls, with some overlap (Figures 1f and 1i). However, with the reduced background signal from the homebrew mixture, the presence of the population of amplicons is now more easily identifiable. Equipped with this homebrew PCR mixture, and an understanding on how to discern the presence of an amplicon population, we next turned to testing of clinical samples. 


\section{Assay Workflow and Testing of Clinical Samples}

For this study we received clinical samples from residual throat swab specimens submitted to the Eastern Ontario Regional Laboratory Association (EORLA) from de-identified patients at the Children's Hospital of Eastern Ontario (CHEO). These swabs were previously used to diagnose the presence of Group A streptococcus (GAS) bacterial infections in children using conventional laboratory techniques. The results from these clinical tests were used for comparison with the nanopore PCR assay workflow.

Figure 2 is a schematic of the workflow used to amplify a region of the genome of GAS using specially designed primers previously clinically validated by qPCR. ${ }^{38}$ Briefly, throat swabs were collected from patients expressing strep-like symptoms when visiting the Emergency Department at CHEO. The sample was obtained by DNA extraction and purified by centrifugation, before undergoing PCR with the optimized components (see Methods). After PCR, the reaction mixture was diluted, combined with salts for nanopore sensing and run on a nanopore for approximately ten minutes. Each nanopore used for the assay also saw a blank negative control consisting of a template-free homebrew PCR reaction mixture. Following nanopore sensing each set of events were analyzed based on the signal profile produced by the blank to classify the background (blank) population. This classified signal is used to identify a subpopulation located around the expected amplicon translocation profile of a sample. The classified amplicon subpopulation is then compared with the classified signal of the blank with a $t$-test. 


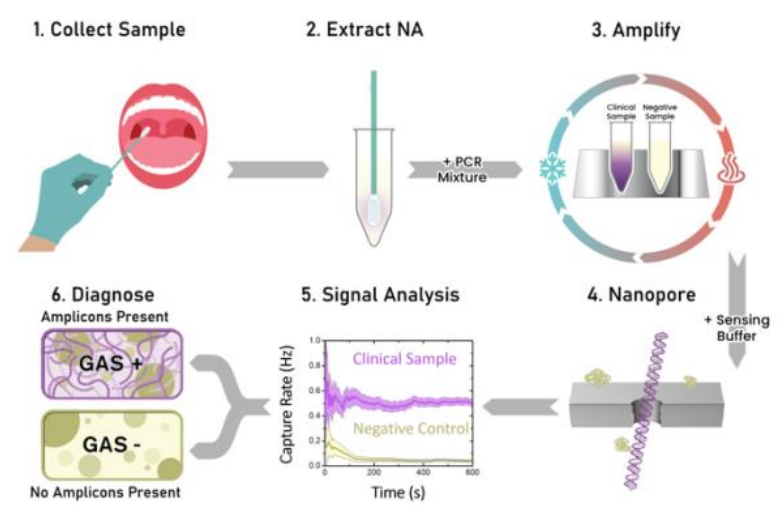

Figure 1: Schematic representation of the assay workflow. Step 1: a throat swab is collected from patients with BD ESwabs. Step 2: DNA is extracted from the throat swab using the Quick-DNA Fungal/Bacterial Miniprep Kit and purified by centrifugation. Step 3: PCR is performed (25 cycles) with a homebrew reaction mixture. Step 4: The sample is mixed with sensing solution and injected in the fluidic flow cell to sense with the nanopore for a few minutes. Step 5: Capture rate of single molecules from sample and blank are compared on the same pore. Step 6: The sample is called GAS positive if a significant amplicon population is present compared to background.

\section{Nanopore Capture Rate-Based Screening}

Here, we analyzed data sets with events from multiple populations of molecules, such as in Figure 11, by a method of population classification based on blockage depth and dwell time. We discuss a few alternative approaches by which such data can be analyzed, and single-molecule events from different population separated, in Supporting Section S4. From the analysis of multiple positive and negative controls, we identified the expected range of blockage depths and dwell times for the amplicon population by Gaussian fitting of the two distributions. This knowledge was then applied the PCR-amplified clinical samples to construct a filter to select all events within an ellipsoid drawn on a log-passage time versus blockage depth plot with each major axis spanning $2 \mathrm{SD}$ about the expected amplicon peak on an individual pore basis, and to calculate the capture rate of events so selected (the "signal rate") as shown in Supporting Figure S8.

To demonstrate the utility of solid-state nanopores as the sensing element in a screening test for the presence of Group A streptococcus (GAS) in throat swabs, we used the assay workflow 
of Figure 2 and compared on the same nanopore the results of a clinical sample with a negative control containing only the PCR mixture. Both sample and control underwent PCR amplification and nanopore sensing under identical conditions. Because even the homebrew PCR mixture produces some events that overlap with the amplicon population, the single-molecule event detection rate from the subpopulation classified as amplicon was used to determine whether the PCR amplification process had generated enough amplicons in the clinical sample to exceed the rate at which signals were generated in the negative control. If a statistically significant increase in the amplicon-classified signal rate over the background was identified from the same population clusters, a clinical sample was then identified as positive. This significance was measured at a twotailed confidence interval of $95 \%$.

If more precise quantitative analysis is desired, we have previously demonstrated an applicable technique (controlled counting) that makes use of a known-concentration standard to account for variations between nanopore and over time in the measured capture rate of a target molecule. ${ }^{39}$ For the purposes of our proof-of-concept study, which focuses simply on screening for the presence or absence of pathogenic DNA, the accuracy and precision of the capture rate data is not as important as qualitatively identifying the GAS-positive samples that produced a clear signal over the negative samples. 
To assess the performance of our assay and analysis workflow on clinical samples, four throat swab samples (two GAS-positive, two GAS-negative) were assessed. The positivity/negativity of the samples for GAS was determined by conventional plating technique (i.e. bacterial culture), and verified by ddPCR. Overall, the data reported in the main text comprises $>10,000$ single-molecule events from 11 experiments acquired on 3 nanopores. The results of these GAS screening tests are presented in Figure 3.

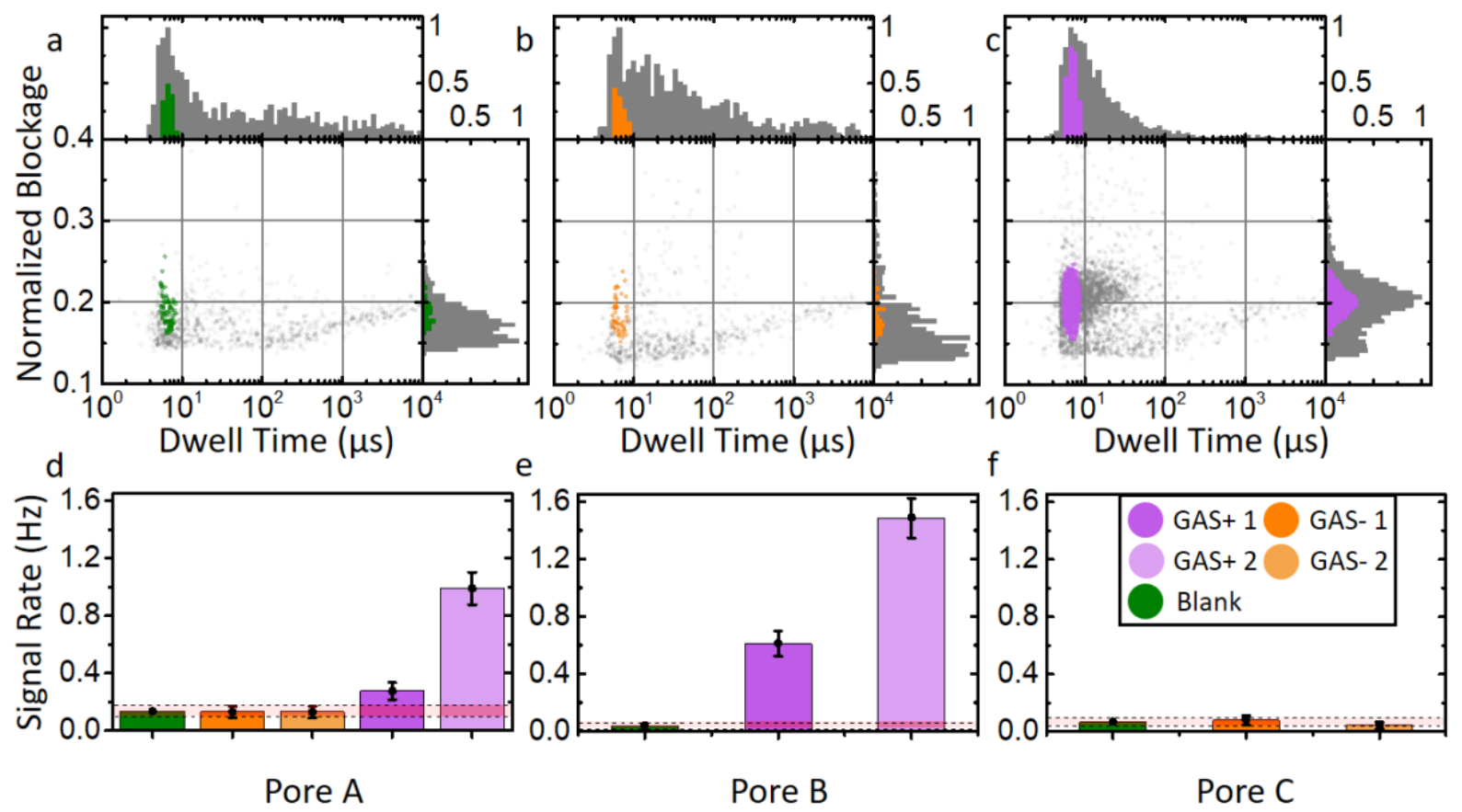

Figure 3: Nanopore analysis of clinical samples. a-c) Blockage fraction as a function of open-pore current vs passage time of all (gray) and amplicon-classified (green, orange, purple) events collected over ten minutes on the same pore with a) blank control ( $\mathrm{n}=816$ total, $\mathrm{n}=72$ classified events), b) GAS-negative ( $\mathrm{n}=662$ total, $\mathrm{n}=47$ classified events), and c) GAS-positive $(\mathrm{n}=2,598$ total, $\mathrm{n}=591$ classified events) samples amplified by PCR. d-f) Signal rate of amplicon-classified events after $300 \mathrm{~s}$ of recording for each sample. Red area bounded by dashed lines represents the $95 \%$ confidence interval of the Blank signal rate. All GAS-neg sample signal rates fall within the blank CI, while GAS-pos sample signal rates exceed the blank CI. Data acquired on pore A ( $6 \pm 1 \mathrm{~nm}$ dia.), pore B (5 $\pm 1 \mathrm{~nm}$ dia.) and pore C (7 $\pm 1 \mathrm{~nm}$ dia.) at 200 $\mathrm{mV}$ in $3.6 \mathrm{M} \mathrm{LiCl} \mathrm{pH8.}$

Figures 3a-c depict the normalized blockage depth and dwell time for all events as well as the classified signals for different samples collected for $\sim 10$ minutes on the same pore. The same 
2 SD criteria, to classify the amplicon population, is applied on all samples on the same pore, though the centroid of the ellipsoid and the actual value of the SD varies between pores. These classified events are shown in color (green, orange, purple) over the entire data set (grey). Analysis of additional clinical samples can be found in Supporting Section S5. The classified signal rate is presented in Figure 3d-f, which shows the capture rates after $300 \mathrm{~s}$ of recording is enough to correctly identify the sample with a $95 \%$ confidence interval. The measurements on each sample were repeated on at least two nanopores to verify reproducibility and consistency of results of our classification approach. The results are summarized in Table 1.

Table 1: Outcomes of all samples tested with the nanopore assay along with initial copy numbers as determined by ddPCR. The signal rate scales with the initial copy number for the same given pore.

\begin{tabular}{|l|l|c|c|}
\hline \multicolumn{1}{|c|}{ Pore ID } & Sample type & $\begin{array}{c}\text { Initial Copy } \\
\text { Number }(\# / \mu \mathbf{L})\end{array}$ & $\begin{array}{c}\text { Signal Rate } \\
(\mathbf{H z})\end{array}$ \\
\hline Pore A & Blank & n/a & $0.14 \pm 0.04$ \\
\hline Pore A & GAS- 1 & $0.7 \pm 0.9$ & $0.13 \pm 0.04$ \\
\hline Pore A & GAS- 2 & undetectable & $0.13 \pm 0.04$ \\
\hline Pore A & GAS+ 1 & $180 \pm 20$ & $0.28 \pm 0.06$ \\
\hline Pore A & GAS+ 2 & $1400 \pm 100$ & $1.0 \pm 0.1$ \\
\hline Pore B & Blank & n/a & $0.05 \pm 0.02$ \\
\hline Pore B & GAS+ 1 & $180 \pm 20$ & $0.56 \pm 0.09$ \\
\hline Pore B & GAS+ 2 & $1400 \pm 100$ & $1.4 \pm 0.1$ \\
\hline Pore C & Blank & n/a & $0.07 \pm 0.03$ \\
\hline Pore C & GAS- 1 & $0.7 \pm 0.9$ & $0.08 \pm 0.03$ \\
\hline Pore C & GAS- 2 & undetectable & $0.04 \pm 0.02$ \\
\hline
\end{tabular}

Variations in the capture rate between the negative samples and blank are reduced to statistical noise when taking the classified signal detection rate, while the positive samples maintain their statistically higher signal rate. Demonstration of the validity of simple capture rate averaging over 
time can be found in Supporting Section S6. Note that the diagnosis results from all three pores are consistent, even if the capture rates for the three pores presented in Table 1 show some variation from pore to pore, which is expected despites being closely related in size, ranging from $5 \pm 1 \mathrm{~nm}$ to $7 \pm 1 \mathrm{~nm}$, and being used under otherwise identical operating conditions. ${ }^{39}$

The low signal rate for the GAS-negative clinical samples shows that very few amplicons were generated as compared to blank control samples. The low level of amplification from these GAS-negative clinical samples could be explained by the presence of the extremely low target concentration (here estimated to be $<1$ copy per $\mu \mathrm{L}$ ) not picked up by bacterial culture (not surprisingly, PCR is more sensitive than conventional plating techniques) or from nonspecific binding of the primers to other genomic DNA present in the throat swab sample. From this limit of blank, we can estimate a limit of detection for this homebrew PCR assay with a few minutes of downstream detection with a single nanopore down to seconds for a small array of tens of pores. The time-evolution of the capture rate shown in Supporting Figure S12

The nanopore screening results agree well with cell cultures and ddPCR techniques. Due to the difference in components and technique between PCR and ddPCR, a direct quantitative comparison between amplicon-classified capture rate and starting copy number is not possible, though the same trend of higher capture rates for higher starting copy numbers was observed. Using the technique presented here, we can make a confident call on the presence or absence of strep-A in a clinical throat swab sample after 25 cycles of PCR with our homebrew reaction mixture, using a purely electrical nanopore readout that obviates the need for optical labelling of the resulting amplicons. 


\section{CONCLUSION}

We have demonstrated that the reagent mixture for PCR can easily be adapted for nanopore sensing, to avoid clogging and spurious events that can prevent measurements or complicate data analysis. We have also provided convincing proof of concept results that nanopores are suitable as the readout mechanism for nucleic-acid amplification-based assay.

Going forward, it will also be interesting to investigate isothermal methods such as NEAR, RPA or LAMP, ${ }^{40-42}$ and to build real-time detection and analysis into the assay to reduce the timeto-answer. While thermocycling comes with requirements for temperature control that most likely precludes real-time sensing of the amplification reaction because of the constantly changing pore conductance, changes in capture, higher, etc., isothermal techniques will likely allow for nanopore measurements in real time, permitting the time to answer for amplification tests assayed with nanopores to match or potentially event exceed fluorescence-based readouts, given that the PCR cycle threshold $\mathrm{C}_{\mathrm{T}}$ can be lower when paired with a nanopore single-molecule detection than it is with optical instrumentation. ${ }^{14}$

While our results show that solid-state nanopores can find utility in point-of-care diagnostics, they also demonstrate clearly that bringing this test into the clinical space requires practical technology development to scale up and parallelize the sensing modality beyond what is currently possible. We hope that these results, and the inspiring proof of concept demonstrations being performed by others in the diagnostics space with solid-state nanopores, will motivate this essential next step in realizing the full potential of solid-state nanopore sensors. 


\section{EXPERIMENTAL}

\section{Sample Collection and Preparation}

Samples were collected with BD ESwabs (BD, 220245) on children being tested for GAS at the Emergency Department of the Children's Hospital of Eastern Ontario (CHEO). DNA was extracted from throat swabs using the Quick-DNA Fungal/Bacterial Miniprep Kit (Zymo D6005) following kit instructions.

\section{Gel Benchmarking}

$2 \%$ agarose (w/v) gel was made in 1X TE buffer with Sybr Safe DNA Gel Stain (Thermofisher, S33102). $5 \mu \mathrm{L}$ sample aliquots were diluted in 6X DNA Gel Loading Dye (Thermofisher, R0611) with water.

\section{Commercial PCR}

Commercial PCR was performed using Thermofisher's PowerUP Sybr Green Master Mix (A25741) according to manufacturer-provided directions for "Standard Cycling Mode (primer $\mathrm{T}_{\mathrm{m}}$ $<60^{\circ} \mathrm{C}$ )" over 25 cycles instead of the instructed 40 cycles.

\section{Nanopore Optimized PCR}

Nanopore optimized PCR was performed using a customized master mix. A 50 $\mathrm{L}$ preparation of nanopore-optimized PCR master mix contained $5 \mu \mathrm{L}$ 10X Taq Buffer (NEB, B9014S), $0.25 \mu \mathrm{L}$ Taq Polymerase (NEB, M0273S), $2.5 \mu \mathrm{L} 10 \mu \mathrm{M}$ DNAse B Forward Primer $(0.5 \mu \mathrm{M}$ - in final volume) [IDT 5'-AGT TGA TTC CAA GAG CTG TCG TG-3'], $2.5 \mu \mathrm{L} 10 \mu \mathrm{M}$ DNAse B Reverse Primer $(0.5 \mu \mathrm{M})$ [IDT 5'-TTA ATG GTG TAG CCA TTA GCT GTG TT-3'], $1 \mu \mathrm{L}$ dNTP mix (NEB, N0447S) $5 \mu \mathrm{L} 10 \mathrm{mM} \mathrm{MgCl} 2(2.5 \mathrm{mM})$, and $31.75 \mu \mathrm{L}$ Nuclease-free ultrapure water 
(Thermofisher, 10977015). $2 \mu \mathrm{L}$ target DNA extract was incubated in this mixture and thermocycled. Starting from $60 \mathrm{~s}$ at $95^{\circ} \mathrm{C}$, the mixture was subjected to 25 cycles of $\left[15 \mathrm{~s}\right.$ at $95^{\circ} \mathrm{C}$, $15 \mathrm{~s}$ at $55^{\circ} \mathrm{C}$, and $30 \mathrm{~s}$ at $68^{\circ} \mathrm{C}$ ], followed by a cooldown $60^{\circ} \mathrm{C}$ for $420 \mathrm{~s}$ then reduction to $4^{\circ} \mathrm{C}$ to stabilize until use.

\section{Nanopore Fabrication}

Nanopores are fabricated using controlled breakdown, ${ }^{43}$ employing the equipment and protocols outlined in detail in Waugh et al. ${ }^{44}$ Briefly, pores were fabricated in nominally $12 \mathrm{~nm}$ thick $\mathrm{SiN}_{\mathrm{x}}$ membranes purchased from Norcada Inc. (NBPX5004Z-60O-Hi RES) in $1 \mathrm{M} \mathrm{KCl} \mathrm{pH} 8$ using a linear voltage ramp, followed by conditioning and enlargement in $3.6 \mathrm{M} \mathrm{LiCl} \mathrm{pH} 8$ until a lownoise pore in the size range of 5-8 $\mathrm{nm}$ was reached.

\section{Data Acquisition and Analysis}

Prior to sensing with the nanopore, $28 \mu \mathrm{L}$ of the sample following the PCR amplification step was diluted with $72 \mu \mathrm{L}$ of $3.6 \mathrm{M} \mathrm{LiCl} 10 \mathrm{mM}$ HEPES pH 8. From that sensing mixture, $40 \mu \mathrm{L}$ is used to perform a nanopore experiment. Amplicon signal was verified using NoLimits 100 bp DNA fragments (ThermoFisher, SM1441)

PCR amplicons are sensed in $3.6 \mathrm{M} \mathrm{LiCl} \mathrm{pH} 8$ using a $200 \mathrm{mV}$ voltage bias. Data are acquired using a Chimera VC100 amplifier sampled at 4.17 MHz with a bandwidth of $1 \mathrm{MHz}$ and are software low-pass filtered at $500 \mathrm{kHz}$ prior to analysis. Events are fitted using a custom implementation of the CUSUM+ algorithm. ${ }^{45}$

\section{ASSOCIATED CONTENT}

Supporting Information 
Additional information on the nanopore signals from the commercial PCR mixture; Details of the optimization process of the homebrew PCR mixture, including gel results; Additional current traces of the homebrew PCR mixture; Details of the method employed for classifying the amplicon population; Additional analysis of clinical samples on different pores; Time-evolution of the capture rate during nanopore recording. This material is available free of charge via the Internet at http://pubs.acs.org

\section{AUTHOR INFORMATION}

\section{Corresponding Author}

*tcossa@uottawa.ca

\section{Author Contributions}

VTC and RS conceived the study. RS acquired clinical samples and performed DNA extraction. SK performed all experimental work and analyzed the data. KB wrote the nanopore analysis software. SK wrote the first draft of the manuscript. VTC KB SK designed the experimental protocols. All authors contributed to the final version of the manuscript.

\section{Funding Sources}

The authors would like to acknowledge the support of the Natural Sciences and Engineering Research Council of Canada (NSERC, grant \#CRDPJ 530554-18,) and the Children's Hospital of Eastern Ontario (CHEO) Research Institute for a Research Growth Award. Notes

KB and VTC declare competing financial interest as founders of Northern Nanopore Instruments Inc., a company commercializing nanopore tools and software for research.

\section{ACKNOWLEDGMENT}


The authors would like to thank François-Xavier Campbell-Valois and John Pezacki for fruitful discussions.

\begin{abstract}
ABBREVIATIONS
DNA, deoxyribonucleic acid; PCR, polymerase chain reaction; BSA, bovine serum albumin;

CBD, controlled breakdown; ddPCR, digital droplet PCR;
\end{abstract}

\title{
REFERENCES
}

1. Weile, J. \& Knabbe, C. Current applications and future trends of molecular diagnostics in clinical bacteriology. Analytical and Bioanalytical Chemistry 394, 731-742 (2009).

2. Bissonnette, L. \& Bergeron, M. G. Diagnosing infections - current and anticipated technologies for point-of-care diagnostics and home-based testing. Clinical Microbiology and Infection 16, 1044-1053 (2010).

3. Gonzalez de Castro, D., Clarke, P. A., Al-Lazikani, B. \& Workman, P. Personalized Cancer Medicine: Molecular Diagnostics, Predictive biomarkers, and Drug Resistance. Clinical Pharmacology \& Therapeutics 93, 252-259 (2013).

4. $\mathrm{Hu}, \mathrm{J}$. et al. Advances in paper-based point-of-care diagnostics. Biosensors and Bioelectronics 54, 585-597 (2014).

5. Xue, L. et al. Solid-state nanopore sensors. Nature Reviews Materials 2020 5:12 5, 931-951 (2020).

6. Kelley, S. O. et al. Advancing the speed, sensitivity and accuracy of biomolecular detection using multi-length-scale engineering. Nature Nanotechnology 2014 9:12 9, 969-980 (2014).

7. Singer, A. et al. Nanopore based sequence specific detection of duplex DNA for genomic profiling. Nano Letters 10, 738-742 (2010).

8. Singer, A., Rapireddy, S., Ly, D. H. \& Meller, A. Electronic barcoding of a viral gene at the single-molecule level. Nano Letters 12, 1722-1728 (2012).

9. Morin, T. J. et al. Nanopore-Based Target Sequence Detection. PLOS ONE 11, e0154426 (2016).

10. Sethi, K. et al. Direct Detection of Conserved Viral Sequences and Other Nucleic Acid Motifs with Solid-State Nanopores. ACS Nano 15, 8474-8483 (2021).

11. Beamish, E., Tabard-Cossa, V. \& Godin, M. Digital counting of nucleic acid targets using solidstate nanopores. Nanoscale 12, 17833-17840 (2020).

12. Beamish, E., Tabard-Cossa, V. \& Godin, M. Programmable DNA Nanoswitch Sensing with SolidState Nanopores. ACS Sensors 4, 2458-2464 (2019). 
13. Squires, A. H., Atas, E. \& Meller, A. Genomic pathogen typing using solid-state nanopores. PLoS ONE 10, e0142944 (2015).

14. Rozevsky, Y. et al. Quantification of mRNA Expression Using Single-Molecule Nanopore Sensing. ACS Nano 14, 57 (2020).

15. Cai, S. et al. Single-molecule amplification-free multiplexed detection of circulating microRNA cancer biomarkers from serum. Nature Communications 2021 12:1 12, 1-12 (2021).

16. Schappert SM \& Burt CW. Ambulatory Care Visits to Physician Offices, Hospital Outpatient Departments, and Emergency Departments: United States, 2001-02. Vital and Health Statistics 13, $1-66$ (2006).

17. Brady, M. T., Jackson, M. A. \& Long, S. S. Red Book 2018-2021 Report of the Committee on Infectious Diseases. 2018-2021 (2018).

18. Langlois, D. M. \& Andreae, M. Group A streptococcal infections. Pediatrics in Review vol. 32 423-429 (2011).

19. Church, D. L., Lloyd, T., Larios, O. \& Gregson, D. B. Evaluation of Simplexa Group A Strep Direct Kit compared to hologic group a streptococcal direct assay for detection of group a streptococcus in throat swabs. Journal of Clinical Microbiology 56, (2018).

20. Davenport, M. et al. The Role of Pore Geometry in Single Nanoparticle Detection. ACS Nano 6, 8366-8380 (2012).

21. Ermann, N. et al. Promoting single-file DNA translocations through nanopores using electroosmotic flow. The Journal of Chemical Physics 149, 163311 (2018).

22. Verschueren, D. v., Jonsson, M. P. \& Dekker, C. Temperature dependence of DNA translocations through solid-state nanopores. Nanotechnology 26, 234004 (2015).

23. Kowalczyk, S. W., Wells, D. B., Aksimentiev, A. \& Dekker, C. Slowing down DNA translocation through a nanopore in lithium chloride. Nano Letters 12, 1038 (2012).

24. Carlsen, A. T. \& Cossa, V. T. Mapping shifts in nanopore signal to changes in protein and proteinDNA conformation. bioRxiv 2020.04.01.020420 (2021) doi:10.1101/2020.04.01.020420.

25. Rosenstein, J. K., Wanunu, M., Merchant, C. A., Drndic, M. \& Shepard, K. L. Integrated nanopore sensing platform with sub-microsecond temporal resolution. Nature Methods 2012 9:5 9, 487-492 (2012).

26. Firnkes, M., Pedone, D., Knezevic, J., Döblinger, M. \& Rant, U. Electrically Facilitated Translocations of Proteins through Silicon Nitride Nanopores: Conjoint and Competitive Action of Diffusion, Electrophoresis, and Electroosmosis. Nano Letters 10, 2162-2167 (2010).

27. Plesa, C. et al. Fast Translocation of Proteins through Solid State Nanopores. Nano Letters 13, 658-663 (2013).

28. Yusko, E. C. et al. Controlling protein translocation through nanopores with bio-inspired fluid walls. Nature Nanotechnology 2011 6:4 6, 253-260 (2011).

29. Garibyan, L. \& Avashia, N. Polymerase Chain Reaction. Journal of Investigative Dermatology 133, 1-4 (2013). 
30. Saiki, R. et al. Primer-directed enzymatic amplification of DNA with a thermostable DNA polymerase. Science 239, 487-491 (1988).

31. Saiki, R. K. et al. Enzymatic amplification of $\beta$-globin genomic sequences and restriction site analysis for diagnosis of sickle cell anemia. Science 230, 1350-1354 (1985).

32. Fologea, D., Ledden, B., McNabb, D. S. \& Li, J. Electrical characterization of protein molecules by a solid-state nanopore. Applied physics letters 91, 053901-1 (2007).

33. Li, W. et al. Single Protein Molecule Detection by Glass Nanopores. ACS Nano 7, 4129-4134 (2013).

34. Bell, N. A. W. \& Keyser, U. F. Specific Protein Detection Using Designed DNA Carriers and Nanopores. Journal of the American Chemical Society 137, 2035-2041 (2015).

35. Gruszka, D., Schmid, S. \& Dekker, C. Nanopores: a versatile tool to study protein dynamics. Essays in Biochemistry 65, 93-107 (2021).

36. Carlsen, A. T., Briggs, K., Hall, A. R. \& Tabard-Cossa, V. Solid-state nanopore localization by controlled breakdown of selectively thinned membranes. doi:10.1088/1361-6528/aa564d.

37. Madejski, G. R. et al. Monolithic Fabrication of NPN/SiNx Dual Membrane Cavity for NanoporeBased DNA Sensing. Advanced Materials Interfaces 6, 1900684 (2019).

38. Slinger, R. et al. Rapid PCR detection of group a streptococcus from flocked throat swabs: A retrospective clinical study. Annals of Clinical Microbiology and Antimicrobials 2011 10:1 10, 15 (2011).

39. Charron, M., Briggs, K., King, S., Waugh, M. \& Tabard-Cossa, V. Precise DNA Concentration Measurements with Nanopores by Controlled Counting. Analytical Chemistry 91, 12228-12237 (2019).

40. Qian, C., Wang, R., Wu, H., Ji, F. \& Wu, J. Nicking enzyme-assisted amplification (NEAA) technology and its applications: A review. Analytica Chimica Acta 1050, 1-15 (2019).

41. Piepenburg, O., Williams, C. H., Stemple, D. L. \& Armes, N. A. DNA Detection Using Recombination Proteins. PLOS Biology 4, e204 (2006).

42. Wong, Y.-P., Othman, S., Lau, Y.-L., Radu, S. \& Chee, H.-Y. Loop-mediated isothermal amplification (LAMP): a versatile technique for detection of micro-organisms. Journal of Applied Microbiology 124, 626-643 (2018).

43. Kwok, H., Briggs, K. \& Tabard-Cossa, V. Nanopore Fabrication by Controlled Dielectric Breakdown. PLoS ONE 9, e92880 (2014).

44. Waugh, M. et al. Solid-state nanopore fabrication by automated controlled breakdown. Nature Protocols 15, 122-143 (2019).

45. Briggs, K. CUSUM v3.3.3 Nanopore Analysis Software Suite. (2020) doi:10.5281/zenodo.595675. 